\title{
Avaliação de aprendizagem em disciplinas de curso técnico a distância
}

\author{
Maria Fernanda Borges-Ferreira \\ Empresa Brasileira de Pesquisa Agropecuária - Embrapa/DF \\ Gardênia Abbad \\ Universidade de Brasília
}

\begin{abstract}
Resumo
Este artigo relata a avaliação de um curso a distância e a análise do relacionamento entre estratégias de aprendizagem, características demográficas, reação ao curso e aprendizagem. Participaram da pesquisa cerca de 2.368 estudantes, que responderam questionários através do ambiente virtual do curso, ao final de cada disciplina. Os participantes possuíam, em média, 19,4 anos de idade $(D P=5,24)$, residiam na região Centro-Oeste $(87,8 \%)$ e pertenciam ao sexo masculino $(77,1 \%)$. Foram testados três modelos de regressão múltipla padrão com aprendizagem como variável critério, medida em termos das notas dos estudantes em atividades avaliativas realizadas ao longo e ao final de cada disciplina. Os resultados mostraram que idade, localização da escola, auto-avaliação de aprendizagem, reações aos procedimentos instrucionais e aos resultados do curso explicaram uma pequena, mas estatisticamente significativa porção da variabilidade das médias finais de aprendizagem.
\end{abstract}

Palavras-chave: avaliação de curso a distância; medidas de aprendizagem; reações ao curso.

\begin{abstract}
Learning evaluation of technical distance course. The present paper aims to report results from an analysis of the relationship among course reaction, learning strategies, demographic characteristic and learning in a distance course context. Approximately 2,368 students constituted the sample of this research. At the end of each lecture, all of them responded to questionnaires hosted at school virtual environment. The students that participated in this study were 19.4 years old $(S D=5.24)$, were located in the center-west region $(87.8 \%)$ and were males $(77.1 \%)$. Three multiple regression analyses were made with learning as the criteria variable. Learning was measured as student's average in evaluation exercises and tests taken during the course and at the end of it. The results showed that age, school location, learning self-evaluation, instructional design reactions and course results reactions explained a small but statistically significant portion of the variability of learning. At the end of this paper, contributions and limitations of this research are discussed.
\end{abstract}

Keywords: distance training evaluation; learning measures; course reactions.

$\mathrm{P}$ rofissionais de diversas áreas, estudantes e empresas têm sofrido o impacto das constantes inovações tecnológicas e da abundância de informações, bem como das mudanças no mundo do trabalho, que exigem perfis profissionais cada vez mais complexos. Diante disso, o indivíduo busca qualificação permanente e aprimoramento de suas competências a fim de lutar contra a obsolescência profissional em favor de sua inserção no mercado de trabalho e da manutenção de sua empregabilidade. As novas tecnologias de informação e comunicação, neste contexto, constituem-se em importantes aliadas na oferta de cursos a distância voltados à qualificação e formação profissionais.

Apesar da importância da educação a distância na qualificação para o trabalho, há poucos estudos sobre os resultados de eventos educacionais ofertados nessa modalidade. Por outro lado, há muita produção de conhecimentos sobre a efetividade de treinamentos presenciais em ambientes organizacionais. Os estudos dessa área investigam as variáveis que predizem resultados de treinamento, entre as quais variáveis referentes às características da clientela, à qualidade da instrução e ao contexto organizacional. Os resultados são medidos em termos de reação ao curso (opiniões ou satisfação do aluno com diversos aspectos de um treinamento), aprendizagem (rendimento do participante), impacto do treinamento no trabalho (transferência de aprendizagem e impacto no desempenho do egresso), mudança organizacional e valor final do treinamento para a organização. Nesses estudos, em geral, os pesquisadores aplicam e validam instrumentos de medida dos diferentes níveis de avaliação.

Este artigo relata a avaliação de disciplinas de um curso técnico profissionalizante a distância, da área de informática, 
no nível de aprendizagem do participante. A pesquisa objetivou analisar o efeito exercido por variáveis relativas às características da clientela (estratégias de aprendizagem, dados demográficos), e reação dos participantes à disciplina sobre aprendizagem (rendimento do aluno).

\section{Breve revisão da literatura e modelo de investigação}

Atividades de avaliação de treinamento representam um importante papel no sistema proposto por Borges-Andrade (1982), uma vez que são as principais responsáveis pelo levantamento de informações avaliativas e retroalimentações para as etapas de levantamento de necessidades, planejamento e execução de treinamento. Kirkpatrick (1976) e Hamblin (1978) propõem modelo de avaliação de treinamento com variáveis relacionadas a resultado de treinamento: reação ao curso (satisfação com a ação instrucional), aprendizagem (assimilação dos conteúdos ou alcance dos objetivos pelo aluno), comportamento no cargo (impacto de treinamento no trabalho), mudança organizacional (efeitos sobre a eficiência de processos e resultados organizacionais) e valor final (efeitos sobre a efetividade organizacional). Segundo os autores, as variáveis mantêm entre si um vínculo correlacional positivo. Alguns estudos, no entanto, apontam para relações fracas ou ausentes entre os níveis.

O Modelo de Avaliação Integrada e Somativa (MAIS), desenvolvido por Borges-Andrade (1982, 2006), surge como alternativa aos modelos anteriormente apresentados. Ele amplia as variáveis de estudo ao acrescentar, aos níveis tradicionais de resultado, variáveis de processo e contexto como variáveis preditivas dos resultados de eventos instrucionais. Os componentes desse modelo são insumo, processo, procedimento, resultado e ambiente (necessidades, apoio, disseminação e resultados a longo prazo).

Outro modelo de avaliação de treinamento é o IMPACT - Modelo Integrado de Avaliação do Impacto do Treinamento no Trabalho (Abbad, 1999), que busca investigar a influência de diferentes variáveis preditoras dos níveis de reação, aprendizagem e impacto e analisar as relações entre os três níveis. Esse modelo tem como base o MAIS e é formado por sete componentes: percepção de suporte organizacional, características de treinamento, características da clientela, reação, aprendizagem, suporte à transferência e impacto do treinamento no trabalho.

Mesmo com a existência de distintos modelos teóricos, verifica-se que a produção científica em avaliação de treinamento, na modalidade a distância, é escassa (Abbad, Carvalho \& Zerbini, 2006; Aguinis \& Kraiger, 2009; Salas \& Cannon-Bowers, 2001). Há carência de estudos que avaliem os efeitos de treinamento dessa natureza, com base nos modelos teóricos dos autores supracitados, bem como que investiguem variáveis explicativas para cada nível avaliado. Nesse sentido, pesquisas voltadas à produção de conhecimentos sobre as variáveis explicativas da eficácia de cursos a distância parecem úteis e necessárias.

Esta pesquisa avaliou disciplinas de um curso técnico profissionalizante, portanto, relacionada à formação profissional de mão-de-obra. Dessa forma, o trabalho relaciona-se mais com os conceitos de formação profissional do que propriamente com o de treinamento, uma vez que o processo de ensino-aprendizagem, em questão, cria oportunidades para que o aluno se qualifique para trabalhos futuros.

Neste estudo são utilizados o MAIS e o IMPACT, modelos adotados mais comumente em avaliações de treinamentos em ambientes organizacionais e de trabalho, porém igualmente aplicáveis em avaliações de cursos em outros contextos de formação, qualificação ou educação, uma vez que ambos têm origem em outros modelos de avaliação, aplicáveis ao contexto educacional como o CIPP (Contexto, Insumo, Processo e Produto) de Stufflebeam (1978) e Hamblin (1978). Além disso, segundo Gagné (1988), eventos instrucionais e de aprendizagem são similares nos diferentes tipos de instrução.

Alguns estudos realizados em contexto de cursos a distância, foram, provavelmente, pioneiros na utilização de modelos que integram diferentes níveis de avaliação e que buscam explicações para cada um desses níveis em variáveis relacionadas ao treinamento, ao contexto organizacional e às características da clientela (Carvalho, 2003; Coelho Júnior, 2004; Zerbini, 2003). Nesses três estudos, o foco principal foi o impacto de treinamento no trabalho.

Segundo Borges-Andrade (2002), um grande desafio para os pesquisadores da área refere-se à avaliação de aprendizagem. Para Abbad e Borges-Andrade (2004), a aprendizagem é um processo psicológico relacionado às mudanças que ocorrem no indivíduo em função de sua relação com o meio. Para esses autores o conceito de aprendizagem, em ambientes de trabalho, inclui os seguintes processos e resultados: aquisição de conhecimentos, habilidades e atitudes; retenção (memorização), generalização (aplicação dos conhecimentos, habilidades ou atitudes em situações diferentes das ensinadas no treinamento), transferência de treinamento (uso, no trabalho, do que foi aprendido no evento instrucional). A aprendizagem em contextos profissionais é valorizada pelo quanto propicia aplicabilidade dos conhecimentos, habilidades e atitudes no trabalho. A aprendizagem ocorre de maneira informal (por meio do contato com pares, chefias) ou formal (por meio de programas ou ações de Treinamento, Desenvolvimento e Educação).

Os maiores avanços na construção de medidas de avaliação de treinamento encontram-se nos níveis de reação ao curso e de comportamento no cargo. Borges-Andrade (2002) salienta que a dificuldade de desenvolvimento de medida no nível de aprendizagem refere-se aos parâmetros de comparação para avaliação, uma vez que, em muitos casos, os treinamentos não apresentam a descrição de seus objetivos. Essa ausência impacta a construção dos itens de avaliação, que, muitas vezes, resultam inválidos e pouco representativos dos comportamentos esperados do participante ao final do curso. Medidas de aprendizagem, quando existentes, são comumente mensuradas por meio de escores específicos obtidos pelos participantes em exercícios, testes de retenção de conhecimentos, ou por meio de médias finais que representam o desempenho ou o rendimento do participante ao final do treinamento. Em treinamentos presenciais, Abbad (1999) analisou modelos de avaliação com os níveis de reação ao curso, aprendizagem e impacto de treinamento no trabalho. A autora encontrou que a origem institucional e o cargo do ocupante tiveram maior influência sobre a aprendizagem (escores 
em pré e pós-testes ou provas de conhecimentos aplicados ao final do treinamento) do que variáveis como reação ao curso. Identificou ainda relações negativas entre idade e aprendizagem e positivas entre motivação para aprender e aprendizagem. Além disso, os participantes com melhor rendimento nos cursos foram também aqueles os que atribuíram a si próprios as melhores auto-avaliações de aprendizagem.

Os resultados de Tannenbaum, Mathieu, Salas e CannonBowers (1991) mostraram que gênero explicou porção significativa das notas obtidas em pós-testes de aprendizagem realizados em treinamentos militares presenciais. Esses autores avaliaram o relacionamento entre características da clientela (dados demográficos, comprometimento, auto-eficácia, motivação e desejos e expectativas do treinando), reações ao curso e aprendizagem.

Pantoja (1999) investigou possíveis preditores de aprendizagem entre variáveis relacionadas às características individuais do treinando, à conduta de entrada em testes de seleção de pessoal, ao treinamento e ao suporte à transferência de treinamento. Nesse estudo, aprendizagem referia-se ao grau de assimilação e à retenção dos conteúdos ensinados no curso, a qual era medida em termos de avaliações práticas (desempenho) e teóricas (conteúdo) realizadas ao final do programa de treinamento.

Os resultados mostraram que a clareza e a precisão na formulação de objetivos instrucionais (correlação positiva) e o estabelecimento de avaliações (correlação negativa), explicaram porção significativa da variabilidade das avaliações teóricas, juntamente com o cargo do aluno. Para a avaliação prática como variável critério, cargo foi negativamente associado à aprendizagem e dois aspectos relacionados à conduta de entrada (notas na entrevista e na prova prática de seleção) estavam positivamente relacionados à aprendizagem.

Em estudo de avaliação de treinamento a distância, Carvalho (2003) tentou encontrar variáveis explicativas para aprendizagem a partir de características da clientela (dados demográficos e uso de ferramentas da web disponibilizadas no curso) e de medidas de reação ao curso (desempenho do tutor, interface gráfica, resultados e aplicabilidade). A variável aprendizagem foi medida em termos do número de acertos nos exercícios de fixação obrigatórios, aplicados ao final de cada etapa do curso. A autora encontrou apenas fraca correlação entre aprendizagem e uso de ferramentas da web e reação aos resultados e aplicabilidade do curso. Esse resultado pode, em parte, ser devido ao tipo de medida de aprendizagem, que exigia a realização dos exercícios, mas não o acerto para avançar no curso, além de ser de um nível de domínio muito baixo, conforme a taxonomia de Bloom (1972, citado por Abbad \& Borges-Andrade, 2004). Apesar do baixo poder de explicação do modelo de avaliação de aprendizagem de Carvalho (2003), um dos resultados estatisticamente significativos dessa pesquisa indicou que os participantes que obtiveram melhores escores de aprendizagem foram os que mais acessaram os chats e o ambiente virtual do curso.

Abbad (1999) e Pantoja (1999) reconhecem a fragilidade das medidas uma vez que não tiveram acesso ao processo de construção dos itens de avaliação. Carvalho (2003) indica a necessidade de desenvolver medidas mais adequadas de aprendizagem a fim de relacioná-las com variáveis da clientela e reação ao curso. A fragilidade das medidas pode ter diminuído a validade preditiva e a fidedignidade e, portanto, a capacidade explicar maior proporção da variância de aprendizagem.

Nos estudos apresentados até o momento, a porcentagem de variância explicada dos modelos de regressão múltipla com aprendizagem é muito baixa. Percebe-se, portanto, a necessidade de medir aprendizagem por meio de instrumentos válidos e confiáveis, baseados nos objetivos instrucionais do curso, de forma a minimizar o erro de medida da avaliação. Para isso, é preciso maior controle sobre a elaboração e aplicação das avaliações. A construção de medidas de aprendizagem deve levar em conta aspectos como modalidade, recursos disponíveis, perfil dos participantes, múltiplas fontes de avaliação, uso de itens de teste variados e padronização dos instrumentos (Abbad, BorgesFerreira, \& Nogueira, 2006).

Abbad, Pilati e Pantoja (2003) relatam que é necessário investir em estudos sobre a influência de características motivacionais e demográficas da clientela, bem como estratégias de aprendizagem sobre os resultados de treinamento, em especial aprendizagem. Os autores verificaram que, nos estudos nacionais, essa categoria tem sido pouco pesquisada. Warr e Downing (2000) encontraram que o uso de estratégias de aprendizagem pelo aluno está relacionado com a aprendizagem.

Diante do exposto, percebe-se a necessidade de investigar variáveis preditoras de aprendizagem, como características da clientela e reação ao curso. Assim, o presente estudo foi estruturado para atender essa demanda.

A medida de aprendizagem foi definida como o rendimento dos alunos no conjunto de atividades avaliativas aplicadas ao longo do curso. Reação ao curso, neste estudo, refere-se à satisfação do participante com aspectos relacionados aos procedimentos instrucionais, ao desempenho da tutoria, aos resultados e à interface gráfica (usabilidade e navegabilidade do ambiente eletrônico do curso).

O componente características da clientela divide-se em dois: dados demográficos e estratégias de aprendizagem. Os dados demográficos referem-se a idade, gênero, escola na qual o aluno está matriculado e região geográfica de residência do participante. As estratégias de aprendizagem, por sua vez, dizem respeito às capacidades cognitivas e habilidades comportamentais, utilizadas pelo aprendiz para controlar os próprios processos psicológicos subjacentes à aprendizagem. De acordo com Warr e Downing (2000), as estratégias possuem as seguintes dimensões: (1) Busca de Ajuda Interpessoal (estratégias utilizadas pelo indivíduo para obter ajuda de colegas e tutores para tirar dúvidas sobre o conteúdo do curso, bem como aprofundar os conhecimentos sobre o assunto discutido no curso); (2) Elaboração e Aplicação Prática (procedimentos adotados pelo indivíduo para verificar possíveis associações entre os conteúdos do curso e seus conhecimentos e experiências práticas anteriores, bem como as suas tentativas de aplicação prática do que foi aprendido); e (3) Repetição, Organização e Ajuda do Material (repetição mental do conteúdo, tal como foi apresentado no treinamento, delimitação das idéias centrais do material e criação de esquemas mentais nos quais os elementos aprendidos são agrupados e relacionados, e obtenção de 
informações em documentos escritos e em outras fontes que não envolvam contato com outros indivíduos).

\section{Método}

A presente pesquisa foi realizada no Centro de Educação Tecnológica MSD Escola Aberta com disciplinas de cursos técnicos profissionalizantes da área de informática. $\mathrm{O}$ material do curso foi veiculado por meio de mídia impressa, CD-ROM e Internet. $\mathrm{O}$ curso foi auto-instrucional, com apoio de central de tutoria on-line e a presença de monitores em escolas conveniadas, as quais disponibilizaram computadores para realização das atividades.

\section{Coleta de dados}

Foram utilizados cinco instrumentos de reação ao curso e de estratégias de aprendizagem. Esses instrumentos foram digitalizados, hospedados na página da instituição e auto- aplicados ao final da disciplina. A cada nova disciplina, o aluno atribuía uma nota para o seu nível de aprendizagem e respondia um dos conjuntos de instrumentos (conjunto 1 Reação à Interface Gráfica, aos Procedimentos Instrucionais e aos Resultados; conjunto 2 - Reação à Tutoria e aos Resultados; e conjunto 3 - Estratégias de Aprendizagem, Reação aos Procedimentos Instrucionais e aos Resultados). O ideal seria a aplicação conjunta de todos os instrumentos, porém, em função das negociações com a Escola, isso não foi possível.

Os itens dos instrumentos estavam associados a uma escala do tipo Likert de 10 pontos, em que 1 (um) era o ponto mínimo e 10 (dez), o máximo. As escalas foram submetidas a análises fatoriais para validação. Todos apresentaram bons índices de qualidade psicométrica (ver Borges-Ferreira, 2005). A Tabela 1 apresenta a estrutura empírica dessas escalas, com número de itens, cargas fatoriais, índice de consistência interna (Alfa de Cronbach), valor próprio (Eigenvalue) e percentual de variância explicada.

Tabela 1

Perfil dos instrumentos de avaliação utilizados na pesquisa após realização de análises fatoriais exploratórias

\begin{tabular}{|c|c|c|c|c|c|}
\hline Instrumento & $\begin{array}{c}\text { № } \\
\text { itens }\end{array}$ & $\begin{array}{l}\text { Cargas } \\
\text { fatoriais }\end{array}$ & $\begin{array}{c}\text { Alfa de } \\
\text { Cronbach }\end{array}$ & $\begin{array}{l}\text { Valor } \\
\text { próprio }\end{array}$ & $\begin{array}{l}\text { Variância } \\
\text { explicada }\end{array}$ \\
\hline Reação à Interface Gráfica & 15 & 0,65 a 0,73 & 0,93 & 7,82 & 52,13 \\
\hline Reação ao Desempenho do Tutor & 28 & 0,50 a 0,85 & 0,97 & 16,02 & 57,21 \\
\hline Reação aos Procedimentos Instrucionais & 12 & 0,52 a 0,73 & 0,89 & 5,59 & 46,59 \\
\hline Reação aos Resultados & 3 & 0,75 a 0,84 & 0,84 & 2,27 & 75,51 \\
\hline \multicolumn{6}{|l|}{ Estratégias de Aprendizagem } \\
\hline Busca de Ajuda Interpessoal & 6 & 0,44 a 0,89 & 0,87 & 5,68 & 29,91 \\
\hline Elaboração e Aplicação Prática & 5 & 0,36 a 0,84 & 0,76 & 1,99 & 10,45 \\
\hline Repetição, Organização e Ajuda do Material & 7 & 0,33 a 0,75 & 0,80 & 0,94 & 4,93 \\
\hline
\end{tabular}

As avaliações de aprendizagem foram construídas pela escola com base nos objetivos de cada disciplina e analisadas por equipe de especialistas em avaliação. Elas eram realizadas de forma sistemática e contínua por meio de atividades e eventos ao longo das disciplinas. Relacionavam-se as seguintes atividades avaliativas: (1) exploração do material didático pelo aluno; (2) exercícios de fixação e aprofundamento dos conteúdos estudados no curso; (3) exercícios práticos com situaçõesproblema relacionados ao trabalho do técnico em informática, com correção pelo tutor disciplina; (4) participação nos fóruns, debates e aulas virtuais realizadas pela Central de Tutoria; e (5) avaliação on-line presencial ao final do curso. Vale ressaltar que, em função da diversidade de avaliações, praticamente todos os objetivos instrucionais foram contemplados. Neste estudo, as três primeiras notas foram denominadas notas baseadas em desempenho e a última, nota baseada em conteúdo. Os dados pessoais (idade, sexo e região geográfica) foram fornecidos automaticamente pelo sistema da Escola.

\section{Característica das disciplinas}

Nas disciplinas analisadas, os objetivos específicos eram, em sua maioria, descritos em termos de desempenhos observáveis, referindo-se à descrição do comportamento esperado do aluno ao final do curso. O conteúdo era apresentado em seqüência lógica e os exercícios e as estratégias utilizados eram compatíveis com a natureza e o nível de complexidade dos objetivos. Além disso, eram disponibilizados recursos de interação mediados pela web.

\section{Amostra}

Os participantes da pesquisa $(N=2368)$ cursaram 43 disciplinas ofertadas durante o curso e representam aproximadamente $60 \%$ dos alunos matriculados entre 2003 e 2004 na Escola. Possuíam, em média, 19,4 anos de idade (DP $=5,24)$, residiam na região Centro-Oeste $(87,8 \%)$ e pertenciam predominantemente ao sexo masculino $(77,1 \%)$. Os alunos estavam matriculados em cursos técnicos da MSD $(90,4 \%)$ e grande parte $(49,2 \%)$ matriculou-se nos cursos via convênio MSD/SENAI-DF.-

\section{Procedimento de análise de dados}

As respostas numéricas dos participantes aos questionários 
foram pareadas e organizadas em arquivo único, de modo a possibilitar a realização de análises de regressão múltipla padrão. Para a execução dessa análise, utilizou-se o Programa Estatístico SPSS (Statistical Package for Social Sciences), versão 11.0.

Antes de testar o modelo, foram feitas análises descritivas e exploratórias para investigar a exatidão da entrada dos dados, a presença de casos extremos, a distribuição dos casos omissos, a distribuição de freqüências e o tamanho das amostras. Foram também testados os pressupostos necessários à realização da análise de regressão múltipla (Tabachnick \& Fidell, 2000).

\section{Resultados}

Nesta seção são relatados os resultados dos três modelos submetidos à análise de regressão múltipla padrão com a variável critério aprendizagem. É importante salientar que a definição de três modelos para o estudo deveu-se à impossibilidade de aplicação do conjunto total de instrumentos a todos os alunos concluintes de disciplinas do curso em análise. Assim sendo, é necessário interpretar os dados para cada modelo em separado.

No primeiro modelo de regressão múltipla com a média final de aprendizagem como variável critério, foram incluídas as seguintes variáveis antecedentes: médias das respostas dos participantes à escala de reação aos resultados, à escala de reação dos participantes ao desempenho da tutoria, idade, sexo, escola, região de origem e auto-avaliação do participante. Ao todo, os dados submetidos a essa análise continham 170 casos válidos.

A Tabela 2 mostra as correlações entre as variáveis do modelo 1, a constante, os coeficientes padronizados de regressão $(\beta)$, as correlações semiparciais $\left(s r^{2}\right)$, bem como o $R, R^{2}$ e o $R^{2}$ ajustado.

Tabela 2

Modelo 1: regressão múltipla padrão para Aprendizagem como variável critério

\begin{tabular}{|c|c|c|c|}
\hline Variável & Aprendizagem & Escola $^{a}$ & $\begin{array}{l}\text { Auto- } \\
\text { Avaliação }\end{array}$ \\
\hline Escola & 0,16 & & \\
\hline $\begin{array}{l}\text { Auto- } \\
\text { Avaliação }\end{array}$ & 0,22 & $-0,05$ & \\
\hline$\beta$ & & $0,24 * *$ & $0,35 * *$ \\
\hline$s r^{2}$ & & 0,20 & 0,28 \\
\hline$X$ & 8,50 & - & 8,05 \\
\hline$D P$ & 0,68 & - & 1,00 \\
\hline \multicolumn{4}{|c|}{$\begin{array}{l}\text { Constante }=7,292 ; R^{2}=0,116^{*} ; R^{2}(\text { ajustado })=0,077^{*} ; \\
\quad R=0,341^{*}\end{array}$} \\
\hline \multicolumn{4}{|c|}{$\begin{array}{l}\text { a em Escola não há média nem desvio-padrão, pois a variável é } \\
\text { dicotômica }\end{array}$} \\
\hline \multicolumn{4}{|c|}{$* * p<0,01 ; * p<0,05$} \\
\hline
\end{tabular}

Apenas duas das sete variáveis incluídas na análise de regressão múltipla contribuíram significativamente para a explicação da variabilidade de aprendizagem: auto-avaliação $\left(s r^{2}=0,28\right)$ e escola na qual o participante fez o curso $\left(s r^{2}=\right.$ $0,20)$. Essas variáveis explicaram aproximadamente $11,6 \%$ de variabilidade da aprendizagem. Isso significa que os alunos com maiores médias finais em aprendizagem foram os que fizeram auto-avaliação mais favorável de sua participação na disciplina e estavam matriculados nas escolas do Estado de Goiás. As menores notas na média final estiveram associadas aos alunos que realizaram as disciplinas em escolas localizadas do Distrito Federal.

No modelo 2 foram incluídas como antecedentes da média final de aprendizagem (variável critério) as seguintes variáveis: reação à interface gráfica, reação aos procedimentos instrucionais, reação aos resultados, idade, sexo, escola, região e auto-avaliação. O arquivo de dados era composto de 677 casos válidos.

A Tabela 3 mostra as correlações entre as variáveis do modelo 2 , a constante, os coeficientes padronizados de regressão $(\beta)$, as correlações semiparciais $\left(s r^{2}\right), \mathrm{o} R, \mathrm{o} R^{2}$ e o $R^{2}$ ajustado.

Tabela 3

Modelo 2: regressão múltipla padrão para Aprendizagem como variável critério

\begin{tabular}{lcc}
\hline \multicolumn{1}{c}{ Variável } & Aprendizagem & $\begin{array}{c}\text { Procedimentos } \\
\text { Instrucionais }\end{array}$ \\
\hline $\begin{array}{l}\text { Procedimentos } \\
\text { Instrucionais }\end{array}$ & $-0,04$ \\
\hline$\beta$ & & $-0,16^{*}$ \\
$s r^{2}$ & 8,55 & $-0,10$ \\
$X$ & 0,59 & 8,71 \\
$D P$ & 0,98 \\
\hline Constante $=8,405 ; R^{2}=0,023^{*} ; R^{2}$ (ajustado) $=0,011^{*} ;$ \\
$R=0,152^{*}$
\end{tabular}

Neste modelo, apenas reação aos procedimentos instrucionais $\left(s r^{2}=-0,10\right)$ contribuiu significativamente para a predição da variável aprendizagem (2,3\%). Portanto, obtiveram maior média final, os participantes que avaliaram menos favoravelmente os procedimentos instrucionais das disciplinas cursadas.

O último modelo foi testado com 10 variáveis antecedentes: médias das respostas dos participantes às escalas de reação aos resultados, reação aos procedimentos instrucionais, médias das respostas dos participantes a três escalas de estratégias de aprendizagem (busca de ajuda interpessoal, estratégias de elaboração e aplicação prática e estratégias de repetição, organização e ajuda do material), idade, sexo, escola, região e auto-avaliação. Foram utilizados 836 casos válidos para realizar a análise de regressão múltipla padrão.

A Tabela 4 mostra as correlações entre as variáveis do terceiro modelo, a constante, os coeficientes padronizados de regressão $(\beta)$, as correlações semiparciais $\left(s r^{2}\right)$, bem como o $R$, $R^{2}$ e o $R^{2}$ ajustado. 
Tabela 4

Modelo 3: regressão múltipla padrão para Aprendizagem como variável critério

\begin{tabular}{|c|c|c|c|}
\hline Variável & Aprendizagem & Resultados & Idade \\
\hline Resultados & 0,11 & & \\
\hline Idade & 0,08 & 0,02 & \\
\hline$\beta$ & & $0,134 * *$ & $0,09 *$ \\
\hline$s r^{2}$ & & 0,095 & 0,09 \\
\hline$X$ & 8,62 & 8,643 & 19,14 \\
\hline$D P$ & 0,59 & 1,246 & 5,03 \\
\hline $\begin{array}{r}\text { Constante }= \\
R=0,18\end{array}$ & $; R^{2}=0,035^{*}$ & (ajustado) & \\
\hline
\end{tabular}

Entre as variáveis incluídas no modelo, apenas reação aos resultados $\left(s r^{2}=0,10\right)$ e idade $\left(s r^{2}=0,09\right)$ contribuíram significativamente para a predição da variabilidade das médias de aprendizagem obtidas pelos participantes nas disciplinas e explicaram $3,5 \%$ de sua variabilidade. Isso significa que obtiveram maior média final os alunos mais novos e os que reagiram mais favoravelmente aos resultados das disciplinas.

\section{Discussão}

A idade entrou como variável preditora de aprendizagem (modelo 3), apresentando relação positiva. Em alguns estudos, aprendizagem esteve negativamente relacionada à idade (Abbad, 1999; Warr \& Bunce, 1995). Abbad (1999), por exemplo, analisou os dados de servidores de organização pública, em sua maioria, com idade entre 31 e 40 anos, que participaram de cursos de diversas áreas. Warr e Bunce (1995) avaliaram gerentes com idade média de 36,08 anos $(D P=8,38)$. Mesmo diferentes na direção do relacionamento, os resultados encontrados pelos autores se assemelham. Nos dois casos, os indivíduos mais jovens eram os que apresentavam maiores escores de aprendizagem. Entretanto, esses indivíduos pertenciam a faixas etárias bem distintas da amostra estudada nesta pesquisa.

Em estudos de avaliação de treinamento a distância, os resultados de Carvalho (2003) mostraram que idade não esteve relacionada à aprendizagem. Vale ressaltar que a amostra estudada pela autora apresentava idade média de 33 anos (DP $=9$ ) e com maior variabilidade do que a encontrada no presente estudo.

Nesta pesquisa, a variável sexo não entrou como explicativa de aprendizagem em nenhum dos modelos analisados, diferentemente do exposto por Tannenbaum et al. (1991), que indicaram gênero do participante como responsável pela explicação de uma parcela significativa das notas nos pós-testes de aprendizagem. No entanto, os autores trabalharam em contexto diferente do investigado por Borges-Ferreira (2005), avaliando recrutas da escola naval norte-americana que participaram de um curso militar introdutório e presencial.

Nenhum fator de estratégias de aprendizagem entrou como variável explicativa dos modelos de regressão múltipla. Esse resultado difere dos obtidos por Warr e Downing (2000) em contextos de treinamento presencial, no qual estratégias de aprendizagem, com exceção das estratégias de busca de ajuda interpessoal e monitoramento da compreensão (essa última não contemplada neste estudo), mostraram-se positivamente correlacionadas com aprendizagem. É bem provável que as estratégias de aprendizagem adotadas pelo aprendiz em cursos presenciais sejam diferentes das utilizadas em cursos a distância.

De Paula e Silva (2004) não encontrou correlações significativas entre estratégias de aprendizagem e resultados de aprendizagem (notas e conceitos finais) obtidos por alunos de graduação em um curso online. Vale ressaltar que as medidas de aprendizagem utilizadas na pesquisa do referido autor eram aparentemente pouco válidas e precisas. Os exercícios, em sua maioria, mensuravam a retenção de conteúdos menos complexos do que as habilidades intelectuais descritas nos objetivos educacionais da disciplina.

A variável reação aos resultados explicou pequena porção estatisticamente significativa da variabilidade de aprendizagem no modelo 3. Esse resultado é similar ao obtido por Carvalho (2003), que encontrou uma fraca correlação positiva entre aprendizagem e reação dos participantes aos resultados e à aplicabilidade do curso. No entanto, a comparação entre os resultados deve ser cautelosa, uma vez que os instrumentos de medida utilizados nos dois estudos possuem diferenças em relação aos itens, sendo o instrumento de Carvalho (2003) mais abrangente, com itens de aplicabilidade/utilidade do curso.

Borges-Ferreira (2005) encontrou que estratégias de elaboração, aplicação prática, repetição, organização e busca de ajuda do material juntamente com auto-avaliação de aprendizagem explicaram uma porção significativa da variabilidade das reações aos resultados dos cursos. Esse resultado indica um provável relacionamento indireto entre estratégias de aprendizagem e aprendizagem, mediado pela variável reação aos resultados.

Neste trabalho, o local de estudo esteve relacionado aos resultados de aprendizagem dos alunos, de modo que as maiores médias finais foram obtidas por alunos que realizaram o curso em escolas localizadas no estado de Goiás. Uma hipótese para a entrada dessa variável no modelo diz respeito à existência de instrutores nas salas aula de alunos do mencionado estado. Esses instrutores, em tese, deveriam apenas auxiliar os alunos quanto ao uso do computador. Porém, de acordo com alguns tutores da Escola, diversos instrutores assumiram funções de tutoria, já que todos eles possuíam formação em informática, compatível com as disciplinas oferecidas pela instituição. Esse fato pode ter resultado no melhor desempenho dos alunos de Goiás em comparação com os alunos que estudaram em escolas do Distrito Federal.

Dois estudos anteriores relacionaram percepção de ganhos de aprendizagem com reação ao curso (Carswell \& Venkatesh, 2002) e com impacto de treinamento no trabalho (Borges-Andrade, Azevedo, Pereira, Rocha, \& Puente-Palacios, 1999) e verificaram que a primeira é variável preditiva das duas últimas. Esse resultado talvez tenha ocorrido porque os alunos com melhores auto-avaliações foram aqueles que mais se empenharam em estudar no decorrer das disciplinas. Esses alunos, provavelmente, realizaram as atividades 
propostas e, conseqüentemente, obtiveram as maiores notas, independentemente do tipo de medida de aprendizagem adotado. No entanto, seria interessante, em outro estudo, conduzir regressões múltiplas com todas as variáveis de características do treinamento e da clientela em um mesmo modelo de predição de aprendizagem. Dessa forma, seria possível verificar se autoavaliação permaneceria na equação de regressão e qual a sua contribuição para a explicação de aprendizagem.

Já a variável reação aos procedimentos instrucionais manteve correlação negativa com aprendizagem. Esse resultado indica que quanto maior a nota, menor a satisfação com os procedimentos instrucionais. Nota-se, entretanto, que a avaliação de reações dos alunos aos aspectos relacionados a carga horária, material didático, seqüência de apresentação e exercícios, fóruns, chats, links e pesquisa via internet) foram, em média, muito boas, variando de 8,57 a 9,17 (desvio-padrão de 1,13 a 1,59). Isso parece indicar que mesmo as avaliações mais baixas foram favoráveis (pontos mais altos da escala). As altas médias atribuídas aos procedimentos instrucionais parecem confirmar as características observadas na avaliação qualitativa do material didático, quanto à adequação da carga horária, à seqüência do conteúdo e à adequação das ferramentas de interação.

Em contexto presencial, Pantoja (1999) também encontrou relacionamento positivo entre algumas variáveis do planejamento instrucional e aprendizagem teórica (retenção de conteúdos). A clareza e a precisão dos objetivos instrucionais mantiveram relação positiva com aprendizagem, enquanto a quantidade de avaliações estabelecidas apresentou relação negativa com aprendizagem teórica. As notas dos participantes nos pré-testes (entrevistas e prova prática de seleção) estavam positivamente associadas à aprendizagem.

Somente a variável reação aos resultados apresentou correlação positiva com aprendizagem, porém esse relacionamento era fraco. Esses resultados corroboram o que outros autores encontraram com relação ao fraco relacionamento entre aprendizagem e reação (Tannenbaum \& Yukl, 1992) e a correlações negativas entre reação e aprendizagem (Abbad, 1999; Alliger \& Janak, 1989).

As medidas de reação, utilizadas nesta pesquisa, aproximamse da definição de reação-satisfação proposta por Alliger, Tannenbaum, Bennett, Traver e Shotland (1997) e que também encontraram fracas relações entre essa variável e aprendizagem. As relações mais fortes, em outros estudos, ocorreram entre aprendizagem e reações-utilidade, medida não presente neste estudo. Carvalho (2003) utilizou, em sua escala de reação aos resultados, itens de aplicabilidade do curso, que se aproximam da definição de utilidade de Alliger et al. (1997). Os resultados da autora mostraram que reação aos resultados e aplicabilidade explicou porção estatisticamente significativa da variabilidade dos escores de aprendizagem. Dessa forma, mais uma vez parece correto afirmar, como fizeram Alliger e Janak (1989), que é problemático assumir como verdadeiros os pressupostos de Kirkpatrick (1976) de que cada nível de avaliação é causado pelo nível imediatamente anterior ou mantêm correlações fortes e positivas entre si.

Vale comentar ainda a discussão feita por alguns autores (Abbad, 1999; Carvalho, 2003) sobre o tipo de medida usado para avaliar os três primeiros níveis de avaliação propostos por Kirkpatrick (1976) e Hamblin (1978). Nos níveis de reação e impacto de treinamento no trabalho são utilizadas medidas perceptuais sujeitas à contaminação por fatores como a desejabilidade social. Por outro lado, há medidas objetivas de aprendizagem que não levam em consideração apenas a percepção do indivíduo, mas seu desempenho. Em estudos que investigam relações entre três ou dois desses níveis percebese que as relações entre os níveis de reação e comportamento no cargo são mais fortes do que entre reação e aprendizagem (Abbad, 1999; Carvalho, 2003; Pantoja, 1999).

Reação ao desempenho da tutoria foi outra variável que, neste estudo, não explicou a variabilidade da aprendizagem. No entanto, esses resultados não são conclusivos. Acredita-se que esta variável deve continuar fazendo parte dos modelos de avaliação em outros contextos. Mesmo sendo o tutor um importante fator de sucesso em cursos a distância, nota-se que grande parte das funções didáticas do professor ou do tutor estão presentes no material do curso, tal como prescrito por Peters (2001). Porém, ainda não se sabe o quanto o tutor é responsável pela aprendizagem e manutenção do aluno em cursos a distância.

Nesta pesquisa, as análises mostraram que os materiais didáticos foram bem elaborados e com conteúdos apresentados, em sua maioria, de forma clara, direta e objetiva. Assim sendo, o tutor poderia não exercer tanta influência sobre a aprendizagem, mas, sim, sobre a persistência do aluno no curso e a realização de exercícios, atividades e provas.

Faz-se necessário, ainda, salientar que o poder explicativo dos modelos é muito pequeno, sugerindo que outras variáveis, não incluídas neste estudo, explicam a aprendizagem. Além disso, a impossibilidade de aplicação do conjunto dos instrumentos pode ter prejudicado a análise.

\section{Considerações finais}

Em todos os modelos de predição de aprendizagem, os coeficientes de determinação foram baixos. Isso indica a existência de outras variáveis, ausentes neste estudo, que explicam a maior parte da variabilidade de aprendizagem. Em estudos futuros, será necessária a inclusão de novas variáveis explicativas em modelo de avaliação de aprendizagem, tais como motivação para aprender, motivação para transferir a aprendizagem, valor instrumental e utilidade do curso para o aprendiz (Abbad, 1999; Salas \& Cannon-Bowers, 2001).

Uma limitação do estudo está relacionada à inexistência de pré-teste de conhecimentos, inviabilizando a mensuração de eventuais ganhos de aprendizagem e a análise das diferenças entre os dois momentos. Sem pré-teste, torna-se inadequada qualquer afirmação conclusiva sobre relações de causa e efeito entre a participação do aluno no curso e o rendimento nas avaliações de aprendizagem. O ideal seria aplicar um desenho experimental ou quase experimental de pesquisa com pré e pósteste com grupo controle. Esse tipo de delineamento diminuiria as ameaças à validade interna da pesquisa.

Contribuições do estudo podem ser atribuídas primeiramente ao uso de medidas de avaliação de aprendizagem construídas 
com base nos objetivos instrucionais. Essas medidas abrangem praticamente todos os objetivos específicos de cada disciplina, corroborando a análise qualitativa do material didático, que as considerou adequadas às suas exigências e bem formuladas. Os problemas de fragilidade da medida enfrentados por alguns pesquisadores e indicados como responsáveis, em parte, pela baixa explicação dos modelos de aprendizagem (Abbad, 1999; Carvalho, 2003; Pantoja, 1999), provavelmente, não ocorreram neste estudo.

Outro ponto que merece destaque é o uso de análise quantitativa feita a partir dos dados coletados na aplicação dos instrumentos de reação e de estratégias de aprendizagem, e de análises qualitativa dos materiais didáticos e das avaliações das disciplinas, que forneceram importantes subsídios para a contextualização da pesquisa e para a interpretação dos resultados das análises estatísticas multivariadas.

Análises do material didático indicaram que é importante avaliar o material instrucional dos cursos e investir em conteúdos que possam ser aplicados pelo aluno em seu contexto de trabalho. Isso porque, a partir da avaliação desse material, percebeu-se que os mais superficiais e com pouca aplicação prática foram os que obtiveram piores avaliações dos alunos e foram considerados, pelos pesquisadores, os mais cansativos de serem realizados e com pouca relação com a prática. Essas características podem levar ao abandono do curso.

Sugere-se a realização de pesquisas futuras para: aplicar os instrumentos em outros contextos (cursos e clientelas); aprimorar medidas de estratégias de aprendizagem; aplicar o conjunto de instrumentos de medida a todos os participantes, o que possibilitaria o pareamento das variáveis de reação ao curso e da clientela com aprendizagem; verificar se cursos com objetivos gerais pertencentes a domínios diferentes (afetivo, cognitivo e psicomotor) estão relacionados a diferentes preditores de aprendizagem; aplicar escala de desejabilidade social e examinar sua influência sobre a satisfação dos alunos com o curso; relacionar variáveis motivacionais e de auto-eficácia com aprendizagem haja vista o relato de autores sobre auto-eficácia e motivação serem boas preditoras de medidas de aprendizagem e impacto de treinamento no trabalho (Lacerda, 2002; Meneses, 2002; Salas \& Cannon-Bowers, 2001); comparar as avaliações de aprendizagem de evadidos e não evadidos, bem como os dados sobre perfil desses indivíduos e verificar se há diferença nas avaliações e, em caso positivo, a que se deve essa diferença; investigar o poder explicativo de um mesmo conjunto de variáveis em relação às diferentes dimensões de aprendizagem, em especial, aquisição, retenção e generalização, aspectos, segundo Abbad e Borges-Andrade (2004), envolvidos no conceito de aprendizagem; e, por fim, examinar o relacionamento entre os três mais tradicionais níveis de avaliação: reação ao curso, aprendizagem e comportamento no cargo.

\section{Referências}

Abbad, G. (1999). Um modelo integrado de avaliação do impacto do treinamento no trabalho - IMPACT. Tese de doutorado não-publicada, Universidade de Brasília, Brasília.

Abbad, G., \& Borges-Andrade, J. E. (2004). Aprendizagem humana em organizações e trabalho. In J. C. Zanelli, J. E. Borges-Andrade \& A. V. B. Bastos (Orgs.), Psicologia: organizações e trabalho no Brasil (pp. 237-275). Porto Alegre: Artmed.

Abbad, G., Borges-Ferreira, M. F., \& Nogueira, R. S. F. (2006). Medidas de aprendizagem. In J. E. Borges-Andrade, G. S. Abbad \& L. Mourão (Orgs.), Treinamento, desenvolvimento e educação em organizações e trabalho: fundamentos para a gestão de pessoas (pp. 469-488). Porto Alegre: Artmed.

Abbad, G., Carvalho, R. S., \& Zerbini, T. (2006). Evasão em curso via internet: explorando variáveis explicativas. RAE-eletrônica, 5(2), art. 17.

Abbad, G., Pilati, R., \& Pantoja, M. J. (2003). Avaliação de treinamento: análise da literatura e agenda de pesquisa. Revista de Administração USP, 38(3), 181-191.

Aguinis, H., \& Kraiger, K. (2009). Benefits of training and development for individuals, and teams, organizations and society. Annual Review of Psychology, 60, 451-474.

Alliger, G. M., \& Janak, E. A. (1989). Kirpatrick's levels of training criteria: thirty years later. Personnel Psychology, 42(2), 331-342.

Alliger, G. M., Tannenbaum, S. I., Bennett, W. Jr., Traver, H., \& Shotland A. (1997). A meta-analysis of the relations among training criteria. Personnel Psychology, 50, 341-358.

Borges-Andrade, J. E. (1982). Avaliação somativa de sistemas instrucionais: integração de três propostas. Tecnologia Educacional, 11(46), 29-39.

Borges-Andrade, J. E. (2002). Desenvolvimento de medidas em avaliação de treinamento. Estudos de Psicologia, 7(n spe.), 31-43.

Borges-Andrade, J. E. (2006). Avaliação integrada e somativa em TD\&E. In J. E. Borges-Andrade, G. S. Abbad, \& L. Mourão (Orgs.), Treinamento, desenvolvimento e educação em organizações e trabalho: fundamentos para a gestão de pessoas (pp. 343-358). Porto Alegre: Artmed.

Borges-Andrade, J. E., Azevedo, L. P. S., Pereira, M. H. G. G., Rocha, K. C. P., \& Puente-Palacios, K. (1999). Impacto de treinamento no trabalho: o caso do Banco do Brasil [Resumo]. In Sociedade Brasileira de Psicologia (Org.), XXIX Reunião Anual de Psicologia. Resumos e Comunicações Cientificas (pp. 53c-53d). Ribeirão Preto: Autor.

Borges-Ferreira, M. F. (2005). Avaliação de reações e aprendizagem em disciplinas de curso técnico profissionalizante oferecidas a distância. Dissertação de mestrado não-publicada, Universidade de Brasília, Brasília.

Carswell, A. D., \& Venkatesh, V. (2002). Learner outcomes in an asynchronous distance education environment. International Journal of Human-Computer Studies, 56, 475-494.

Carvalho, R. S. (2003). Avaliação de treinamento à distância: reação, suporte à transferência e impacto do treinamento no trabalho. Dissertação de mestrado não-publicada, Universidade de Brasília, Brasília.

Coelho Júnior, F. A. (2004). Avaliação de treinamento à distância: suporte à aprendizagem e impacto do treinamento no trabalho. Dissertação de mestrado não-publicada, Universidade de Brasília, Brasília.

De Paula e Silva, A. (2004). Avaliação de uma disciplina semipresencial de graduação ofertada por meio da internet pela Universidade de Brasília. Dissertação de mestrado não-publicada, Universidade de Brasília, Brasília.

Gagné, R. M. (1988). Mastery learning and instructional design. In R. C. Richey (Org.), The legacy of Robert Gagné (pp. 107-126). Nova York: ERIC Clearinghouse on Information \& Technology e International Board of Standards for Training Performance and Instruction - IBSPTI.

Hamblin, A. C. (1978). Avaliação e controle do treinamento. São Paulo: McGraw-Hill.

Kirkpatrick, D. L. (1976). Evaluation of training. In R. L Craig (Org.), Training and development handbook (pp. 18.1-18.27). Nova York: McGraw-Hill.

Lacerda, E. (2002). Avaliação de impacto do treinamento no trabalho: investigando variáveis motivacionais e organizacionais como suas preditoras. Dissertação de mestrado não-publicada, Universidade de Brasília, Brasília.

Meneses, P. P. M. (2002). Auto-eficácia, locus de controle, suporte à transferência 
e impacto de treinamento no trabalho. Dissertação de mestrado nãopublicada, Universidade de Brasília, Brasília.

Pantoja, M. J. B. (1999). Avaliação de impacto de treinamento na área de reabilitação: preditores individuais e situacionais. Dissertação de mestrado não-publicada, Universidade de Brasília, Brasília.

Peters, O. (2001). Didática do ensino a distância. São Leopoldo: Unisinos.

Salas, E., \& Cannon-Bowers, J. (2001). The science of training: a decade of progress. Annual Review of Psychology, 52, 471-499.

Stufflebeam, D. (1978). Alternativas em avaliação educacional: um guia de autoensino para educadores. In M. Scriven \& D. Stufflebeam (Orgs.), Avaliação Educacional (II): perspectivas, procedimentos e alternativas (pp. 50-150). Petrópolis: Vozes.

Tabachnick, B. G., \& Fidell, L. S. (2000). Using multivariate statistics (4⿳⺈⿴囗十一 Nova York: Harper-Collins.
Tannenbaum, S. I., Mathieu, J. E., Salas E., \& Cannon-Bowers J. A. (1991). Meeting trainees' expectations: the influence of training fulfillment on the development of commitment, self-efficacy, and motivation. Journal of Applied Psychology, 76(6), 759-769.

Tannenbaum, S. I., \& Yukl, G. (1992). Training and development in work organizations. Annual Review of Psychology, 43, 399-441.

Warr, P., \& Bunce, D. (1995). Trainee characteristics and outcomes of open learning. Personnel Psychology, 48, 347-375.

Warr, P., \& Downing, J. (2000). Learning strategies, learning anxiety and knowledge acquisition. British Journal of Psychology, 91, 311-333.

Zerbini, T. (2003). Estratégias de aprendizagem, reações aos procedimentos de um curso via internet, reações ao tutor e impacto do treinamento no trabalho. Dissertação de mestrado não-publicada, Universidade de Brasília, Brasília.

Maria Fernanda Borges-Ferreira, mestre em Psicologia Social e do Trabalho pela Universidade de Brasília, é analista do Departamento de Gestão de Pessoas - DGP da Empresa Brasileira de Pesquisa Agropecuária (Embrapa). Endereço para correspondência: SHIS QI17 conj. 04 casa 05, Brasília, DF; CEP: 71645-040. Telefones: (61) 3248-1575/ 8101-7069.E-mail: mfernandaborges@gmail.com

Gardênia da Silva Abbad, doutora em Psicologia Social e do Trabalho pela Universidade de Brasília, é professora adjunta no Departamento de Psicologia Social e do Trabalho do Instituto de Psicologia da mesma universidade. E-mail: gardenia.abbad@gmail.com 